\title{
Andreev Reflection and Creation of Spin-Entangled Charge Carriers in Double Barrier Junctions
}

\author{
R.J. WOJCIECHOWSKI* AND L. KOWALEWSKI \\ Solid State Theory Division, Faculty of Physics, A. Mickiewicz University \\ Umultowska 85, 61-614 Poznań, Poland
}

\begin{abstract}
We discuss spin dependent single particle charge transport as well as the spin pairwise entanglement processes in a double barrier junction. The junction consists of a superconductor connected to two normal or spin-polarized conductors, or semiconductors. Special attention is focused on a non-local process known as a crossed Andreev reflection. The non-local Andreev reflection occurs when an incident electron (hole) from one lead, injected with a subgap energy onto a superconducting layer, transforms into an Andreev hole (electron) in the second lead. At the same time, a Cooper pair is created in the superconductor. Using the Bogolubov-de Gennes equation with appropriate boundary conditions we calculate and discuss probabilities of processes relevant for charge transport through the junction. The dependence of the tunneling charge transport on the strength and orientation of the exchange field in the ferromagnetic electrodes, and on the height of the tunnel barriers as well as on the distance between the electrodes are presented. We discuss briefly the physics of creation and charge transport of spin entangled electron-hole pairs in the junction.
\end{abstract}

PACS numbers: 74.45.+c, 73.23.Ad, 03.65.Ud

\section{Introduction}

In this paper we have considered charge transport through $\mathrm{M} / \mathrm{S} / \mathrm{M}$ nanoscale junctions ( $\mathrm{M}$ - metal, half-metal, ferromagnetic metal or semiconductor; S - superconductor). In particular, we have concentrated on the role of the local (LAR) and nonlocal (crossed Andreev reflection - CAR) Andreev reflections [1-8] both in the single-particle transport as well as the pairwise spin entangled transport. The charge transport can be decomposed into two components, local if the entangled quasiparticles enter the same lead and nonlocal if they enter different leads. The local processes occur in the single normal- or ferromagnetic (F) metal-superconductor junction. The nonlocal processes occur when the distance $l$ between the electrodes of the $\mathrm{M} / \mathrm{S} / \mathrm{M}$ junction is less than or comparable to the superconducting coherence length $\xi[7,8]$. Experimental evidence for CAR and for the elastic cotunneling (EC) has been given by Russo et al. [3] and by Beckmann and von Löhneysen [4]. If the thickness of $\mathrm{S}$ layer is large enough, a double tunnel junction can be regarded as two independent single $\mathrm{M} / \mathrm{S}$ junctions and then the tunneling processes become incoherent [6]. In addition, the total electronic current in the $\mathrm{F} / \mathrm{S} / \mathrm{F}$ junction strongly depends on the relative orientation of the magnetization of two ferromagnetic electrodes $[7,8]$. CAR is a consequence of capturing an ad-

* corresponding author; e-mail: wojrysz@amu.edu.pl ditional electron (or hole) from the second (e.g. right) electrode in order to form a Cooper pair in the superconductor. The appropriate hole (or electron) is reflected in the right electrode. EC consists in the direct tunneling of the injected particle from one electrode to the second one, without formation of the Cooper pair [5]. The electron-like quasiparticle penetrates depth of order of the superconducting coherence length $\xi$ before its conversion into the condensate of the Cooper pairs. The quasiparticle current $J_{\mathrm{Q}}$ in the superconducting layer disappears, in $y$-direction across the junction, according to the formula $J_{\mathrm{Q}}=2 e v_{\mathrm{F}} \exp (-y / \xi)$, where the coherence length is given by $\xi=\hbar v_{\mathrm{F}} /\left(2 \sqrt{\Delta^{2}-E^{2}}\right), \Delta$ is the superconducting gap. Simultaneously, $J_{\mathrm{Q}}$ is transformed into an increasing supercurrent of the Cooper pairs $J_{\mathrm{s}}=2 e v_{\mathrm{F}}[1-\exp (-y / \xi)]$. In this paper, we discuss how the nonlocal effects are influenced by the energy of the incident electron and the width $l$ of the superconducting layer.

In general, the phase coherent, ballistic charge transport, through the multi-barrier junctions with superconducting layers, may also lead to quantum entanglement of the charge carriers. In particular, in the case of $\mathrm{M} / \mathrm{S} / \mathrm{M}$ junction CAR enables realization of the solid state entanglers, which create mobile and spatially separated entangled pairs of electrons (holes) or an electron-hole pair. In Sect. 3, we discuss briefly the problem of creation and spatial separation of spin entangled electron-hole pairs in the $\mathrm{M} / \mathrm{S} / \mathrm{M}$ junction. 


\section{Model and the calculations}

In this section, we consider a single particle transport in a double junction $\mathrm{M} / \mathrm{S} / \mathrm{M}$, consisting of two metallic (normal or ferromagnetic) electrodes and a superconducting layer separated from the electrodes by thin insulating interfaces. We consider a model of the $\mathrm{M} / \mathrm{S} / \mathrm{M}$ junction with a clean superconductor. In the clean limit, one can easily solve the Bogolubov-de Gennes (BdG) equations in the approach proposed in [9]. The wave function, describing the quasiparticle propagation across the $\mathrm{M} / \mathrm{S} / \mathrm{M}$ junction, can be obtained from extended $\mathrm{BdG}$ equations. In the ferromagnetic regions, these equations are reduced to the appropriate Schrödinger equations of the form

$$
\begin{aligned}
& \left(\begin{array}{cc}
H_{0}-E-\sigma h_{\mathrm{ex}}, & \Delta \\
\Delta^{\dagger} & -\left(H_{0}-E+\sigma h_{\mathrm{ex}}\right)
\end{array}\right)\left(\begin{array}{c}
f_{\sigma}(\boldsymbol{r}) \\
g_{-\sigma}(\boldsymbol{r})
\end{array}\right) \\
& =\hat{O},
\end{aligned}
$$

where $H_{0}=-\hbar^{2} / 2 m \nabla^{2}-E_{\mathrm{F}}+W(\boldsymbol{r})$ is the single-particle Hamiltonian, the quasiparticle energy $E$ is measured from the Fermi energy $E_{\mathrm{F}}$, and $\sigma=+(-)$ denotes the up (down) spin subband. In the case of a normal metal $\sigma=0, W(\boldsymbol{r})$ stands for the interface potential which is expressed by the dimensionless parameter $z=2 m W / \hbar^{2} k_{\mathrm{F}}[5,9]$. For the ferromagnetic electrodes, we adopt the Stoner model. Thus, the exchange field in both the left $(\mathrm{L})(y<-l / 2)$ and the right $(\mathrm{R})$ electrodes $(y>l / 2)$ can be defined as follows:

$$
h_{\mathrm{ex}}= \begin{cases}h_{0}, & y<-l / 2, \\ 0, & -l / 2<y<+l / 2, \\ \pm h_{0}, & y>l / 2,\end{cases}
$$

where $+h_{0}$ and $-h_{0}$ are the exchange fields for the parallel and antiparallel configuration, respectively. The solution of Eq. (1) for the injection of an electron with spin $\sigma$ (from one, e.g. the left ferromagnetic electrode) and with the energy $E$, and the angle of incidence $\Theta$ can be written in the following form:

$$
\begin{aligned}
& \Psi_{\sigma}^{(y<-l / 2)}(y)=\left(\begin{array}{l}
1 \\
0
\end{array}\right) \mathrm{e}^{\mathrm{i} k_{\sigma}^{+} y} \\
& +a_{-\sigma}\left(\begin{array}{l}
0 \\
1
\end{array}\right) \mathrm{e}^{\mathrm{i} k_{-\sigma}^{-} y}+b_{\sigma}\left(\begin{array}{l}
1 \\
0
\end{array}\right) \mathrm{e}^{-\mathrm{i} k_{\sigma}^{+} y}, \\
& \Psi_{\sigma}^{(y>l / 2)}(y)=c_{\sigma}\left(\begin{array}{l}
1 \\
0
\end{array}\right) \mathrm{e}^{\mathrm{i} k_{\sigma(-\sigma)}^{+} y} \\
& +d_{\sigma}\left(\begin{array}{l}
0 \\
1
\end{array}\right) \mathrm{e}^{-\mathrm{i} k_{-\sigma(\sigma)}^{-} y},
\end{aligned}
$$

where the $y$ component of the momentum for both an electron $\left(k_{\sigma}^{+}\right)$and hole $\left(k_{\sigma}^{-}\right)$takes the form: $k_{\sigma}^{ \pm}=\sqrt{\left(2 m / \hbar^{2}\right)\left(E_{\mathrm{F}}+\sigma h_{0} \pm E\right)-\boldsymbol{k}_{\|, \sigma}^{2}}$. The momentum parallel to the interface has the form: $\left|\boldsymbol{k}_{\mid, \sigma}\right|=$ $\sqrt{\left(2 m / \hbar^{2}\right)\left(E_{\mathrm{F}}+\sigma h_{0}+E\right)} \sin (\Theta)$.

The probability amplitudes of the transport processes $a_{\sigma}(E, \Theta), b_{\sigma}(E, \Theta), c_{\sigma}(E, \Theta), d_{\sigma}(E, \Theta)$ determine LAR,

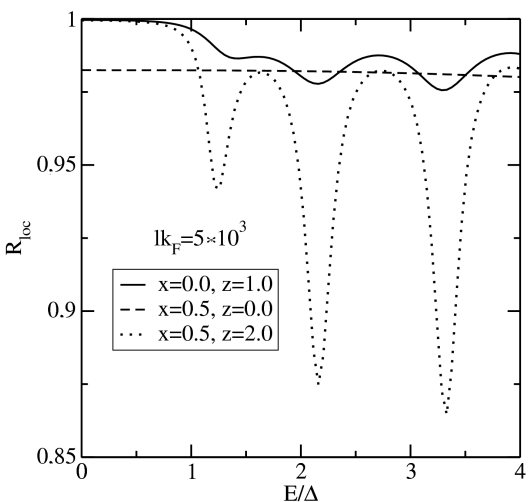

Fig. 1. Relative contribution of the LAR $R_{\text {loc }}=$ $\left|a_{\sigma}\right|^{2} /\left(\left|a_{\sigma}\right|^{2}+\left|d_{\sigma}\right|^{2}\right)$ to the entanglement processes as a function of $E / \Delta$ for several values of $x=h_{0} / E_{\mathrm{F}}$ and $z=2 m W / \hbar^{2} k_{\mathrm{F}}$ and for $l<\xi$.

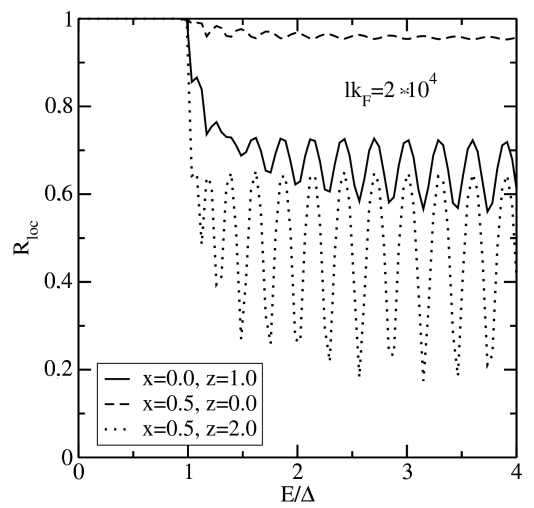

Fig. 2. Relative contribution of LAR $R_{\text {loc }}$ to the transmission processes as a function of $E / \Delta$ for several values of $x$ and $z$ and for $l>\xi$.

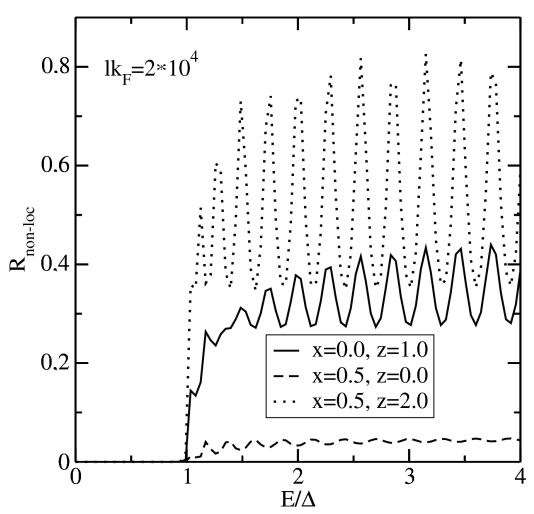

Fig. 3. Relative contribution of CAR $R_{\text {non-loc }}=$ $\left|d_{\sigma}\right|^{2} /\left(\left|a_{\sigma}\right|^{2}+\left|d_{\sigma}\right|^{2}\right)$ to the transmission processes as a function of $E / \Delta$ for several values of $x$ and $z$ and for $l<\xi$. 


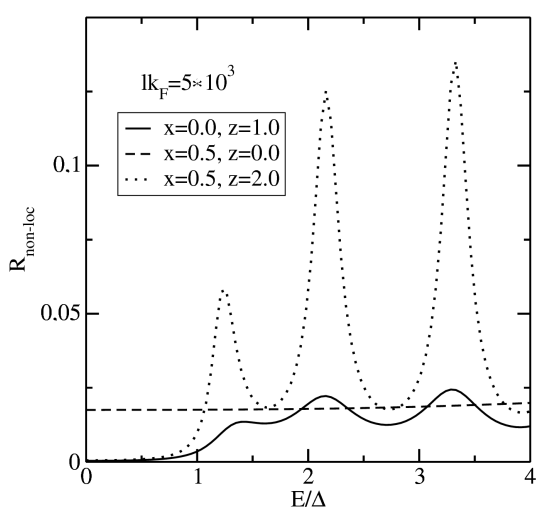

Fig. 4. Relative contribution of CAR $R_{\text {non-loc }}$ to the transmission processes as a function of $E / \Delta$ for several values of $x$ and $z$ and for the width $l>\xi$.

normal (NR) reflections, EC, and CAR, respectively [5-8, $10,11]$. The relative values of the single particle local $R_{\mathrm{loc}}=\left|a_{\sigma}\right|^{2} /\left(\left|a_{\sigma}\right|^{2}+\left|d_{\sigma}\right|^{2}\right)$ and non-local $R_{\text {non-loc }}=$ $\left|d_{\sigma}\right|^{2} /\left(\left|a_{\sigma}\right|^{2}+\left|d_{\sigma}\right|^{2}\right)$ Andreev reflections are presented in Figs. 1-4. The oscillations in Figs. 1-4 are due to the interference of the incoming and outgoing particles in the superconducting layer. The non-local process is completely suppressed for sub-gap energies and $l>\xi$. For $E / \Delta>1$, the probabilities of the coherent single particle charge transport processes (LAR, CAR, NR, and EC) oscillate with the energy and the thickness of the superconducting layer. Moreover, both $a_{\sigma}$ and $d_{\sigma}$ vanish if the following resonance condition is fulfilled: $q_{\sigma}^{+}-q_{\sigma}^{-}=$ $2 n \pi / l$, for the momentum in the superconducting layer $\mathrm{S}$ given by $q_{\sigma}^{ \pm}=\sqrt{\left(2 m / \hbar^{2}\right)\left(E_{\mathrm{F}} \pm \sqrt{E^{2}-\Delta^{2}}\right)-\boldsymbol{k}_{\|, \sigma}^{2}}[5,6$, 10, 11]. The coefficients $a_{\sigma}(E, \Theta), b_{\sigma}(E, \Theta), c_{\sigma}(E, \Theta)$, $d_{\sigma}(E, \Theta)$ are determined by the appropriate boundary conditions for the wave functions and their derivatives at the both interfaces which are placed at $y= \pm l / 2$. These coefficients determine not only the charge transport through $\mathrm{M} / \mathrm{S} / \mathrm{M}$ junctions, but also they reflect the pairing symmetry of the superconductor (see e.g. [12]). The sign of the voltage, measured across the second $\mathrm{S} / \mathrm{M}$ tunnel barrier of the junction $\mathrm{M} / \mathrm{S} / \mathrm{M}$, depends upon whether EC or CAR occurs with larger probability [3]. In the case of half-metallic ferromagnet electrodes $\left(x=h_{0} / E_{\mathrm{F}}=1\right)$ the process of CAR can be completely suppressed while EC is allowed, or vice versa, depending on the mutual (parallel or antiparallel) alignments of the electrode magnetizations. In the case of the singlet superconductors, the processes which lead to tunneling of the particles to the different leads, can be enhanced for the antiparallel magnetic configuration, or can be suppressed for the parallel configuration of the ferromagnetic electrodes. This coherent transport is particularly interesting because of its promising perspective for spintronics application by implementation of magnetoresistive devices, such as spin switches, memory elements, magnetic read-out heads for computer hard drives, spin transistors etc.
The separation between the both, left $(\mathrm{L})$ and right $(\mathrm{R})$ metallic electrodes should be comparable to the superconducting coherence length $(\xi)$ in order to enable CAR.

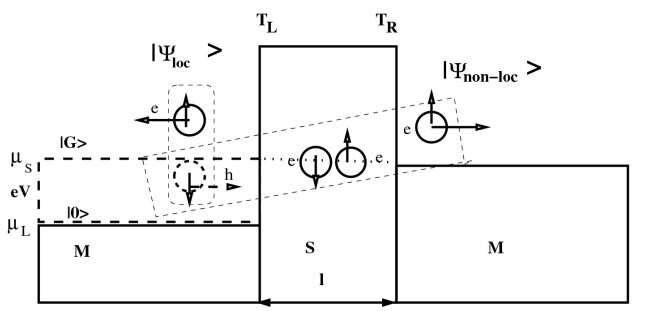

Fig. 5. Sketch of the reflection processes responsible for local and non-local entanglement in the $\mathrm{M} / \mathrm{S} / \mathrm{M}$ junction.

\section{The Andreev entangler: creation of spin entangled pairs}

The charge transport in $\mathrm{M} / \mathrm{S} / \mathrm{M}$ junctions is accompanied by the creation of spin entangled quasiparticle pairs. The source of this entanglement is the Cooper pairing interaction. If a negative (positive) bias voltage $e V=\mu_{\mathrm{S}}-\mu_{\mathrm{L}}$ is applied to the left conductor, then the holes (electrons), incident on the superconducting layer, change their sign of charge and their spin direction (Fig. 5). $R_{\text {loc }}$ and $R_{\text {non-loc }}$ are directly related to the efficiency of the production of the entanglement electronhole pairs. It can be seen in Fig. 4 that if $E>\Delta$, the production of the non-local spin-entangled pairs is more effective for large values of the barrier strength $z$ and $l>\xi$. The ground state of the left electrode $|0\rangle$ is defined as follows by the appropriate hole creation operators:

$$
|0\rangle=\prod_{0<E<e V, \sigma} a_{\mathrm{L} \sigma}^{\mathrm{h} \dagger}(E)|G\rangle,
$$

where $|G\rangle$ denotes the Fermi sea filled up to the superconducting chemical potential $\mu_{\mathrm{s}}$ (see Fig. 5). It is convenient to write the input state $\left|\Psi_{\text {in }}\right\rangle$ of the holes (electrons), injected to the superconducting layer, in terms of the stream of hole (electron) pairs with the opposite spins. For example, for the hole pairs we can write $[13,14]$ :

$$
\left|\Psi_{\text {in }}\right\rangle=\frac{\mathrm{i}}{2} \prod_{0<E<e V} \hat{a}_{\mathrm{L}}^{\mathrm{h} \dagger}(E) \sigma_{y} \hat{a}_{\mathrm{L}}^{\mathrm{h} \dagger \mathrm{T}}(E)|G\rangle,
$$

where $\hat{a}_{\mathrm{L}}^{\mathrm{h}}=\left(a_{\mathrm{L} \uparrow}^{\mathrm{h}}(E) a_{\mathrm{L} \downarrow}^{\mathrm{h}}(E)\right)^{\mathrm{T}}$ is a column matrix of the annihilation operators of a pair of holes with the antiparallel spins and energy $E$ at the left conductor. $\sigma_{y}$ and $T$ stands for the Pauli matrix and the matrix transpose, respectively. The evolution of the input state $\left|\Psi_{\text {in }}\right\rangle$ into the output state $\left|\Psi_{\text {out }}\right\rangle$ can be connected through unitary transformation, which can be expressed in terms of the scattering matrix $\hat{S}[13]$. The scattering matrix matches the wave functions at both $\mathrm{M} / \mathrm{S}$ and $\mathrm{S} / \mathrm{M}$ interfaces in a double barrier junction [15]. In our further discussion, 
we take into account only the Andreev reflections and neglect the EC. A relation between the input $(\hat{a})$ and output $(\hat{b})$ matrices of the electron (e) and hole (h) annihilation operators can be written in the following form [13]:

$$
\hat{b}=\hat{S} \hat{a},
$$

where $\hat{a} \equiv\left(a_{\mathrm{L} \uparrow}^{\mathrm{e}}, a_{\mathrm{L} \downarrow}^{\mathrm{e}}, a_{\mathrm{R} \uparrow}^{\mathrm{e}}, a_{\mathrm{R} \downarrow}^{\mathrm{e}}, a_{\mathrm{L} \uparrow}^{\mathrm{h}}, a_{\mathrm{L} \downarrow}^{\mathrm{h}}, a_{\mathrm{R} \uparrow}^{\mathrm{h}}, a_{\mathrm{R} \downarrow}^{\mathrm{h}}\right)^{\mathrm{T}}$ and $\hat{b} \equiv\left(b_{\mathrm{L} \uparrow}^{\mathrm{e}}, b_{\mathrm{L} \downarrow}^{\mathrm{e}}, b_{\mathrm{R} \uparrow}^{\mathrm{e}}, b_{\mathrm{R} \downarrow}^{\mathrm{e}}, b_{\mathrm{L} \uparrow}^{\mathrm{h}}, b_{\mathrm{L} \downarrow}^{\mathrm{h}}, b_{\mathrm{R} \uparrow}^{\mathrm{h}}, b_{\mathrm{R} \downarrow}^{\mathrm{h}}\right)^{\mathrm{T}}$ are column matrices. $\hat{S}$ is $8 \times 8$ scattering matrix, which describes all the reflection processes at the left $(\mathrm{L})$ and right $(\mathrm{R})$ interfaces:

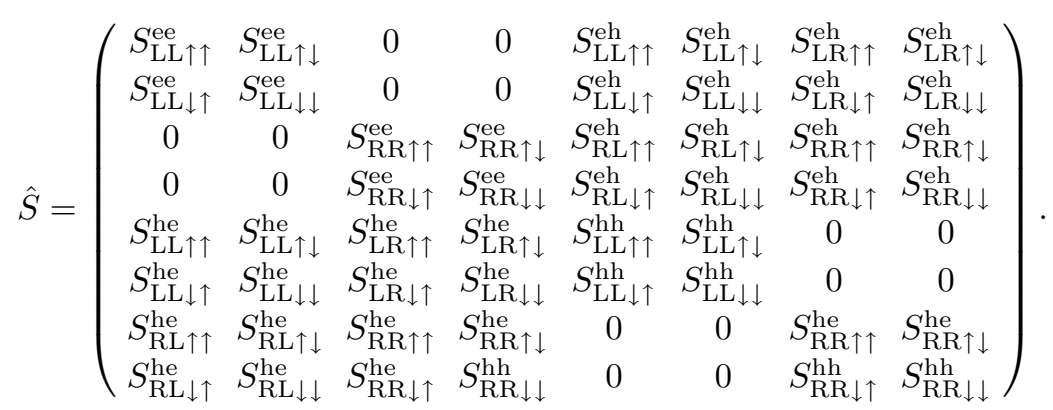

All the elements of the scattering matrix $\hat{S}$ describe appropriate Andreev reflections. For example, $S_{\mathrm{LL} \uparrow \downarrow}^{\mathrm{eh}}$ denotes the LAR amplitude for a hole with spin down injected from the left lead (L) and reflected from the M/S interface, as an electron with spin up into the same lead.

In the tunneling limit, the transparencies of the tunnel barriers $\left(T_{\mathrm{L}(\mathrm{R})}\right)$ as well as the elements of the scattering matrix $\hat{S}$ obey the relations: $T_{\mathrm{L}(\mathrm{R})} \ll 1$ and $S_{(\ldots)}^{\alpha \beta} \ll 1$. Thus, the outgoing state, to the first order of the scattering matrix elements, can be written as a superposition of the vacuum state $|0\rangle$ and the local and non-local spin singlet entangled states [13]:

$$
\begin{aligned}
& \left|\Psi_{\text {out }}\right\rangle=\sqrt{1-T_{\mathrm{L}}^{2}-T_{\mathrm{L}} T_{\mathrm{R}} \gamma(l)}|0\rangle+T_{\mathrm{L}}\left|\Psi_{\text {loc }}\right\rangle \\
& \quad+\sqrt{T_{\mathrm{L}} T_{\mathrm{R}} \gamma(l)}\left|\Psi_{\text {non-loc }}\right\rangle
\end{aligned}
$$

where the local and non-local spin singlet states have the form

$$
\begin{aligned}
& \left|\Psi_{\text {loc }}\right\rangle \\
& \quad=\frac{1}{\sqrt{2}}\left[b_{\mathrm{L} \uparrow}^{\mathrm{h} \dagger}(E) b_{\mathrm{L} \downarrow}^{\mathrm{e} \dagger}(E)-b_{\mathrm{L} \downarrow}^{\mathrm{h} \dagger}(E) b_{\mathrm{L} \uparrow}^{\mathrm{e} \dagger}(E)\right]|0\rangle, \\
& \left|\Psi_{\text {non-loc }}\right\rangle \\
& \quad=\frac{1}{\sqrt{2}}\left[b_{\mathrm{L} \uparrow}^{\mathrm{h} \dagger}(E) b_{\mathrm{R} \downarrow}^{\mathrm{e} \dagger}(E)-b_{\mathrm{L} \downarrow}^{\mathrm{h} \dagger}(E) b_{\mathrm{R} \uparrow}^{\mathrm{e} \dagger}(E)\right]|0\rangle .
\end{aligned}
$$

$\gamma(l)=\exp \left(-\frac{2 l}{\pi \xi}\right)$ describes how a Cooper pair decays with the width $l$ of the superconducting layer. As seen from Eq. (9), the outgoing state consists of the locally $\left(T_{\mathrm{L}}\right)$ and non-locally $\left(\sqrt{T_{\mathrm{L}} T_{\mathrm{R}}}\right)$ spin entangled electronhole pairs. In our case, this spatially separated stream of entangled electron-hole pairs is created by the tunnel barriers in the $\mathrm{S} / \mathrm{M}$ junction due to the superconducting layer. Further numerical calculations, concerning an influence of the oscillations of the transport processes through $\mathrm{M} / \mathrm{S} / \mathrm{M}$ junctions $[5,6,10,11]$, on the charge and spin currents, and on the degree of spin entanglement in these junctions, are in progress.

\section{Results and conclusions}

The coherent charge transport through the double junction $\mathrm{M} / \mathrm{S} / \mathrm{M}$ reveals many unusual features both for the subgap transmission and for energy greater than superconducting gap. It was shown $[5,6,10,11]$ that, if the energy of the incident electron is greater than the superconducting gap $\Delta$, the probabilities of all the components of the coherent transport processes, i.e. LAR, CAR and NR as well as the direct tunneling through the double junction, oscillate as the functions of $E / \Delta$ and $l$ due to the interference of the incoming and outgoing particles in the superconducting layer. If the thickness of the superconductor layer $l$ is smaller than the superconducting coherence length $\xi$, the non-local processes EC and CAR play an important role in the charge transport for the subgap energy. In the opposite limit, suppression of EC and CAR is observed [5, 6, 10]. Observation of the variations of the tunneling current in double junctions for different widths of the superconducting layer is reported in [5]. The maxima (minima) of the elastic cotunneling correspond one to one to minima (maxima) of LAR, CAR, and NR. If $l \geq \xi$, then the non-local processes decay. When EC dominates, i.e. when the charge carriers pass from the left to the right electrode without conversion into the supercurrent, LAR and CAR decay at the both interfaces. It is worthy mentioning that the typical ranges of the coherence length are of the order of $\xi=10$ Aand $\xi=1.0 \mu \mathrm{m}$ for high- $T_{\mathrm{c}}$ and low-temperature superconductors, respectively. The electron-beam lithography technique allows one to fabricate devices which have sizes of the order of tens $\mathrm{nm}$.

The important role in the transport processes through the double junction is played by the pairing symmetry 
of a superconducting state and the magnetic orientation of the ferromagnetic electrodes [12]. For example, in the case of the spin singlet pairing and the parallel magnetic configuration of the electrodes, LAR and CAR are suppressed and for half-metals $\left(x=h_{0} / E_{\mathrm{F}}=1\right)$ these processes are completely blocked. In the case of antiparallel alignment, CAR is favoured while LAR is suppressed (or blocked for half-metals). In Sect. 3 we have briefly discussed another intriguing aspect of the coherent transport in the double barrier junction, where except the single particle transport the spin-entangled electron-hole pairs can tunnel into the local and non-local leads. The spatially separated spin entangled electron-hole pairs are created due to tunnel barriers and CAR. Another source of non-local spin entangled electron pairs is the emission of electrons from an $s$-wave superconductor to two separated leads via quantum dots, in the Coulomb blockade regime (see e.g. [16]).

Multiterminal junctions e.g. $\mathrm{M} / \mathrm{S} / \mathrm{M}$ are promising examples of solid state entanglers. The controlled creation and detection of the mobile entangled pairs of charge carriers, in spatially separated nanoelectronic leads, is crucial for quantum information. The spin of these mobile particles can be used as qubits. The nonlocal spin-entangled electron pairs can be detected e.g. via the current-noise measurements in transport experiments.

Finally, it can be said, for instance, that various aspects of the tunneling quasiparticle transport, mainly the coherent one, through the double barrier junctions exhibits unusual features which turned out to be useful in nanoelectronic physics. The coherent pairwise tunneling transport of spin entangled pairs in spatially separated leads seems to be very promising for quantum information.

\section{References}

[1] J.P. Morten, A. Brataas, W. Belzig, Phys. Rev. B $\mathbf{7 4}$ 214510 (2006).

[2] J.M. Byers, M. Flatté, Phys. Rev. Lett. 74, 306 (1995); Phys. Rev. Lett. 74, 3305 (1995).

[3] S. Russo, M. Kroug, T.M. Klapwijk, A.F. Morpurgo, Phys. Rev. Lett. 95, 027002 (2005); Phys. Rev. Lett. 95, 229901 (2005).

[4] D. Beckmann, H. v. Löhneysen, AIP Conf. Proc. 850, 875 (2006).

[5] J.M. Božević, Z. Radović, Phys. Rev. B 66, 134524 (2002); Phys. Rev. B 71, 229901(E) (2005).

[6] Z.C. Dong, R. Shen, Z.M. Zheng, D.Y. Xing, Z.D. Wang, Phys. Rev. B 67, 134515 (2003).

[7] T. Yamashita, H. Imamura, S. Takahashi, S. Maekawa, Phys. Rev. B 67, 094515 (2003).

[8] T. Yamashita, S. Takahashi, S. Maekawa, Phys. Rev. B 68, 174504 (2003).

[9] G.E. Blonder, M. Tinkham, T.M. Klapwijk, Phys. Rev. B 25, 4515 (1982).

[10] L. Kowalewski, R.J. Wojciechowski, Acta Phys. Pol. A 115, 57 (2009).

[11] R.J. Wojciechowski, L. Kowalewski, J. Phys., Conf. Series, in press.

[12] C. Benjamin, Phys. Rev. B 74, 180503 (2006).

[13] Z. Yeng Zeng, L. Zhou, J. Hong, B. Li, Phys. Rev. 74, 085312 (2006).

[14] C.W.J. Beenakker, C. Emary, M. Kindermann, J.L. van Velsen, Phys. Rev. Lett. 80, 147901 (2003).

[15] C.W.J. Beenakker, Rev. Mod. Phys. 69, 731 (1997).

[16] P. Recher, E.V. Sukhorukov, D. Loss, Phys. Rev. B 63, 165314 (2001). 